Theor Popul Biol. 2012 August ; 82(1): 59-65. doi:10.1016/j.tpb.2012.04.002.

\title{
Ascertainment correction for a population tree via a pruning algorithm for likelihood computation
}

\author{
Arindam RoyChoudhury ${ }^{1}$ and Elizabeth A. Thompson ${ }^{2}$ \\ ${ }^{1}$ Department of Biostatistics, Columbia University, New York NY 10032 \\ ${ }^{2}$ Department of Statistics, University of Washington, Seattle WA 98195
}

\begin{abstract}
We present a method for correcting ascertainment-bias in a coalescent-based likelihood for population trees. Our method is computationally simple and fast. To correct for the bias we compute the probability of allele-counts conditioned on the locus being included. This conditional probability is simply the uncorrected likelihood divided by the inclusion probability. A modification of a pruning algorithm is introduced so that the inclusion probability can be computed with a single run of the algorithm. Our computation is exact and avoids Monte-Carlo based methods.
\end{abstract}

\section{Keywords}

phylogeny; population tree; pruning; ascertainment; likelihood

\section{Introduction}

Ascertainment is the process of selecting observational or experimental units for inclusion in a study. If these units are genetic single nucleotide polymorphism (SNP) loci, several considerations apply.

The SNPs with a small minor allele frequency provide little information for some genetic analyses (see, for example, Clayton [3]). Geneticists may therefore ascertain the sample so that each SNP locus has at least a few copies of the minor allele. Various SNP selection criteria exist in practice, with different criteria affecting the data differently. It is not uncommon for the selection process to use a complicated assortment of criteria. For example, the HapMap project ([4]) uses many pieces of information to identify SNPs (Clark et al. [2]).

\footnotetext{
(c) 2012 Elsevier Inc. All rights reserved.

Corresponding author: Arindam RoyChoudhury, Department of Biostatistics, 722 W 168th St. 6th Floor, New York NY 10032, U.S.A. Phone: 1-212-342-1268, Fax: 1-212-305-9408, ar2946@ columbia.edu.

Publisher's Disclaimer: This is a PDF file of an unedited manuscript that has been accepted for publication. As a service to our customers we are providing this early version of the manuscript. The manuscript will undergo copyediting, typesetting, and review of the resulting proof before it is published in its final citable form. Please note that during the production process errors may be discovered which could affect the content, and all legal disclaimers that apply to the journal pertain.
} 
The ascertainment process distorts the allele frequency spectrum of the SNPs chosen for the study as well as the whole distribution of data outcomes. If the distortion of this distribution is not corrected for, an erroneous inference may result. For example, see, Clark et al. [2], for some illustrations of how ascertainment-bias can affect inference. Also, later in this article we illustrate how failure to correct for ascertainment-bias may introduce bias in the branch length estimates of population tree. Thus, correcting for the effect of ascertainment is a necessary part of a study involving SNP allele frequencies or counts.

Whereas no known analysis (including our method) can fully correct for ascertainmentbias in a data collected using a highly complicated ascertainment strategy like HapMap (see, for example, Clark et al. [2]), using our method one will be able to correct for ascertainmentbias in data with where the ascertainment strategies are known and constant across the loci.

We implement a fast and simple method for correcting ascertainment bias when computing the likelihood of a population tree from SNP allele frequency spectra. The uncorrected likelihood is computed using the pruning algorithm of RoyChoudhury et al. [9].

To correct for the ascertainment bias we need to compute the probability of inclusion; that is, the sum of the probabilities of all outcomes that make up the ascertainment event (see, for example, Nielsen and Slatkin [7]). In the context of phenotypic data on pedigrees Elston [5] and Elston and Yelverton [6] used a pedigree peeling computation to obtain the probability of the ascertainment event. Analogously, in the case of population trees, we use the pruning algorithm of RoyChoudhury et al. [9], to compute the inclusion probability for several commonly used ascertainment strategies. The probability of sample allele counts falling within a specified set can be computed once only with a single application of the pruning algorithm. Thus our algorithm is computationally much faster than an approach which requires computation of the inclusion-probability for each SNP.

\section{Theory and methods}

As in RoyChoudhury et al. [9], we assume that loci are sufficiently far apart that the allele counts are independent and the loci have independent coalescent events. We will use the term "independent loci" to refer to such a set of loci. Consequently, the likelihood from $L$ loci is the product of the likelihoods from each locus. Therefore, we only need to consider the computation of likelihood from a single locus. We also assume that each locus has two alleles, arbitrarily named ' 0 ' and ' 1 '.

As in RoyChoudhury et al. [9], the population-tree is specified by a set of parameters: the branch lengths, the tree-topology and a mutation parameter $\theta_{0}$ at the root. (Note that, we denote the root as node 0 .) The branch lengths are scaled units of generations. We assume, as in RoyChoudhury et al. [9], that the allele frequency spectrum at the root follows a symmetric Beta distribution with $\theta_{0}$ as its sole parameter.

To describe the data and the related latent variables, we will use the notation of RoyChoudhury et. al [9] throughout this paper. For readers' convenience, the notation is given in Table 1 and Figure 1. We term "a location just below a node, in the branch coming from the left (or right) side of the node $x^{\prime \prime}$, to mean a time-point $x^{\prime}$ (on the left branch or the 
right branch coming from the node), that is infinitesimally close to $x$ (so that no coalescent event could have taken place between $x$ and $x^{\prime}$ ).

\subsection{The pruning algorithm}

The pruning algorithm (RoyChoudhury et al. [9]) works by computing two arrays of conditional probabilities at each node. The arrays at each tip are computed from the data at the tip. Then the arrays at each non-tip node $x$ are computed from the nodes directly below $x$ (see Figures 1 and 2). Thus, the arrays for all the nodes are computed, moving upward node by node (see Figure 2). Eventually the arrays at the root are computed, and the likelihood is computed from these arrays.

Two arrays $\mathrm{A}(x)$ and $\mathrm{B}(x)$ are computed at each node $x$ :

$$
\mathrm{A}(x)=\left(\operatorname{Pr}\left(n_{x}=i\right), i=1,2, \ldots, m_{x}^{(\mathrm{b})}\right),
$$

where $n_{x}$ is the number of lineages at node $x$ and $m_{x}^{(\mathrm{b})}$ is the total number of gene copies sampled at or below node $x$;

$$
\mathrm{B}(x)=\left(\left(\operatorname{Pr}\left(s_{x}^{(\mathrm{b})} \mid n_{x}=i, r_{x}=j\right), j=0,1,2, \ldots, i\right), i=1,2, \ldots, m_{x}^{(\mathrm{b})}\right),
$$

where $S_{x}^{(\mathrm{b})}$ is all the data at or below node $x$ and $r_{x}$ is the allele-count (the count of ' 1 ' alleles) out of $n_{x}$ lineages at node $x$. Thus,

$$
\mathrm{A}(x)_{i}=\operatorname{Pr}\left(n_{x}=i\right) ; \quad \mathrm{B}(x)_{i j}=\operatorname{Pr}\left(s_{x}^{(\mathrm{b})} \mid n_{x}=i, r_{x}=j\right)
$$

Two similar arrays $\left(\mathrm{A}^{(\mathrm{L})}(x)\right.$ and $\left.\mathrm{B}^{(\mathrm{L})}(x)\right)$ are also computed for the point just below, and coming from the left side of node $x$ :

$$
\mathrm{A}^{(\mathrm{L})}(x)=\left(\operatorname{Pr}\left(n_{x}^{(\mathrm{L})}=i\right), i=1,2, \ldots, m_{x}^{(\mathrm{b}, \mathrm{L})}\right),
$$

where $n_{x}^{(\mathrm{L})}$ is the number of lineages just below node $x$, in the branch coming from the left side of $x$ and $m_{x}^{(\mathrm{b}, \mathrm{L})}$ is the total number of gene copies sampled at or below the branch coming from the left side of node $x$;

$$
\mathrm{B}^{(\mathrm{L})}(x)=\left(\left(\operatorname{Pr}\left(s_{x}^{(\mathrm{b}, \mathrm{L})} \mid n_{x}^{(\mathrm{L})}=i, r_{x}^{(\mathrm{L})}=j\right), j=0,1,2, \ldots, i\right), i=1,2, \ldots, m_{x}^{(\mathrm{b}, \mathrm{L})}\right),
$$

where $s_{x}^{(\mathrm{b}, \mathrm{L})}$ is all the data at or below the branch coming from the left side of node $x$ and $r_{x}^{(\mathrm{L})}$ is the allele-count (out of $n_{x}^{(\mathrm{L})}$ lineages) just below node $x$, in the branch coming from the left side of $x$. Thus,

$$
\mathrm{A}^{(\mathrm{L})}(x)_{i}=\operatorname{Pr}\left(n_{x}^{(\mathrm{L})}=i\right) ; \quad \mathrm{B}^{(\mathrm{L})}(x)_{i j}=\operatorname{Pr}\left(s_{x}^{(\mathrm{b}, \mathrm{L})} \mid n_{x}^{(\mathrm{L})}=i, r_{x}^{(\mathrm{L})}=j\right)
$$


The arrays $\mathrm{A}^{(\mathrm{R})}(x)$ and $\mathrm{B}^{(\mathrm{R})}(x)$ are similarly computed for each point just below, and coming from the right side of node $x$.

Consider a branch with nodes $z$ and $y$ at its top and bottom respectively. Without loss of generality, assume that the branch is on the left bottom side of $z$. "Step 1" in Figure 2 is the computation of the arrays $\left(\mathrm{A}^{(\mathrm{L})}(z)\right.$ and $\left.\mathrm{B}^{(\mathrm{L})}(z)\right)$ at the top of the branch from the arrays $(\mathrm{A}(y)$ and $\mathrm{B}(y))$ at the bottom of the branch. In "Step 2" we combine the arrays of probabilities $\left(\mathrm{A}^{(\mathrm{L})}(x), \mathrm{B}^{(\mathrm{L})}(x), \mathrm{A}^{(\mathrm{R})}(x)\right.$ and $\left.\mathrm{B}^{(\mathrm{R})}(x)\right)$ at the common top point $x$ of the two branches and thus derive the arrays at $x(\mathrm{~A}(x), \mathrm{B}(x))$. In Appendix $\mathrm{A}$ we state the formulae used by RoyChoudhury et al. [9] for the computations of Steps 1 and 2.

Repeating Steps 1 and 2 (Figure 2), we eventually compute the arrays $\mathrm{A}(0)$ and $\mathrm{B}(0)$ at the root of the tree, and then the likelihood is computed as

$$
\mathrm{L}(\boldsymbol{\tau})=\sum_{i=1}^{m_{0}^{(\mathrm{b})}} \sum_{j=0}^{i} \mathrm{~B}(0)_{i j} \mathrm{~A}(0)_{i}\left(\begin{array}{c}
i \\
j
\end{array}\right)\left(\frac{\beta(j+\theta, i-j+\theta)}{\beta(\theta, \theta)}\right),
$$

where $\tau$ is the vector containing all the branch lengths and $m_{0}^{(\mathrm{b})}$ is the total number of gene copies sampled below the root, equivalently, the total number of gene copies sampled in all the populations.

\subsection{Ascertainment Corrections}

Each ascertainment strategy is characterized by a rejection set $\mathrm{R}$ which is a subset of the set of all possible allele-count matrices, as described below. Suppose that $D_{i 1}$ and $D_{i 2}$ are the numbers of ' 1 ' and ' 0 ' alleles (respectively) observed in population $i$ and

$$
\mathbf{D}=\left(\begin{array}{cc}
D_{11} & D_{12} \\
D_{21} & D_{22} \\
\ldots & \ldots \\
D_{P 1} & D_{P 2}
\end{array}\right)
$$

the matrix of allele-counts from a locus $l$. For example, suppose we have three populations with 20 haploids from each population and the observed number of ' 1 ' alleles are 16,2 and 5 respectively from the three populations. (As in RoyChoudhury et al. [9], haploid is our sampling unit.) Then

$$
\mathbf{D}=\left(\begin{array}{cc}
16 & 4 \\
2 & 18 \\
5 & 15
\end{array}\right)
$$

Let $U$ be the set of all possible values $\mathbf{D}$ can take. Each ascertainment strategy is characterized by a rejection set $R$ (which is a subset of $U$ ) as follows. If $\mathbf{D}$ falls into $R$, then $l$ is excluded. The set $\mathrm{R}^{\mathrm{C}}$, which is the compliment of $\mathrm{R}$, is the acceptance set. If $\mathbf{D}$ falls into $\mathrm{R}^{\mathrm{C}}$, then $l$ is included. 
For an ascertainment based on the current sample, the corrected likelihood from a locus (given that it is included in the study) is

$$
\mathrm{L}(\boldsymbol{\tau} \mid \mathbf{D}=\mathbf{d})=\operatorname{Pr}\left(\mathbf{D}=\mathbf{d} \mid \mathbf{D} \in \mathrm{R}^{\mathrm{C}}\right)=\frac{\operatorname{Pr}(\mathbf{D}=\mathbf{d})}{\operatorname{Pr}\left(\mathbf{D} \in \mathrm{R}^{\mathrm{C}}\right)}
$$

where $\operatorname{Pr}(\mathbf{D}=\mathbf{d})$ is the uncorrected likelihood. Suppose that an ascertainment is done based on a previous sample. Let the matrix of allele-counts in the previous sample be

$$
\mathbf{D}^{(\mathrm{v})}=\left(\begin{array}{cc}
D_{11}^{(\mathrm{v})} & D_{12}^{(\mathrm{v})} \\
D_{21}^{(\mathrm{v})} & D_{22}^{(\mathrm{v})} \\
\ldots & \ldots \\
D_{P 1}^{(\mathrm{v})} & D_{P 2}^{(\mathrm{v})}
\end{array}\right),
$$

where $D_{i 1}^{(\mathrm{v})}$ and $D_{i 2}^{(\mathrm{v})}$ are the numbers of ' 1 ' and ' 0 ' alleles (respectively) observed in population $i$ in the previous sample. Then the correct likelihood is

$$
\begin{aligned}
& \mathrm{L}\left(\boldsymbol{\tau} \mid \mathbf{D}=\mathbf{d}, \mathbf{D}^{(\mathrm{v})}=\mathbf{d}^{(\mathrm{v})}\right) \propto \operatorname{Pr}\left(\mathbf{D}+\mathbf{D}^{(\mathrm{v})}=\mathbf{d}+\mathbf{d}^{(\mathrm{v})} \mid \mathbf{D}^{(\mathrm{v})} \in \mathrm{R}^{\mathrm{C}}\right) \\
& =\quad \frac{\operatorname{Pr}\left(\mathbf{D}+\mathbf{D}^{(\mathrm{v})}=\mathbf{d}+\mathbf{d}^{(\mathrm{v})}\right)}{\operatorname{Pr}\left(\mathbf{D}^{(\mathrm{v})} \in \mathrm{R}^{\mathrm{C}}\right)} \text {. }
\end{aligned}
$$

Eq. 5 follows from the fact that

$$
\frac{\operatorname{Pr}\left(\mathbf{D}+\mathbf{D}^{(\mathrm{v})}=\mathbf{d}+\mathbf{d}^{(\mathrm{v})} \mid \mathbf{D}^{(\mathrm{v})} \in \mathrm{R}^{\mathrm{C}}\right)}{\operatorname{Pr}\left(\mathbf{D}=\mathbf{d}, \mathbf{D}^{(\mathrm{v})}=\mathbf{d}^{(\mathrm{v})} \mid \mathbf{D}^{(\mathrm{v})} \in \mathrm{R}^{\mathrm{C}}\right)}
$$

is free of $\tau$, and thus the probability of observing allele-counts $\mathbf{d}$ and $\mathbf{d}^{(\mathrm{v})}$ in the two samples from $P$ populations is a constant multiple (upto $\tau$ ) of the probability of observing allelecounts $\mathbf{d}+\mathbf{d}^{(\mathrm{v})}$ in a combined sample. Another way of looking at it is that $\operatorname{Pr}\left(\mathbf{D}=\mathbf{d}, \mathbf{D}^{(\mathrm{v})}=\right.$ $\left.\mathbf{d}^{(\mathrm{v})} \mid \mathbf{D}+\mathbf{D}^{(\mathrm{v})}=\mathbf{d}+\mathbf{d}^{(\mathrm{v})}\right)$ is free of $\tau$ and depends only on the sample sizes and allele counts, and thus it can be ignored while computing the likelihood.

If $\mathbf{d}^{(\mathrm{v})}$ is not available, then the corrected likelihood is

$$
\mathrm{L}(\boldsymbol{\tau} \mid \mathbf{D}=\mathbf{d})=\operatorname{Pr}\left(\mathbf{D}=\mathbf{d} \mid \mathbf{D}^{(\mathrm{v})} \in \mathrm{R}^{\mathrm{C}}\right)=\frac{\operatorname{Pr}\left(\mathbf{D}=\mathbf{d}, \mathbf{D}^{(\mathrm{v})} \in \mathrm{R}^{\mathrm{C}}\right)}{\operatorname{Pr}\left(\mathbf{D}^{(\mathrm{v})} \in \mathrm{R}^{\mathrm{C}}\right)} \propto \frac{\operatorname{Pr}\left(\mathbf{D}+\mathbf{D}^{(\mathrm{v})} \in \mathrm{G}\right)}{\operatorname{Pr}\left(\mathbf{D}^{(\mathrm{v})} \in \mathrm{R}^{\mathrm{C}}\right)},
$$

where

$$
\mathrm{G}=\mathbf{d}+\mathrm{R}^{\mathrm{C}}=\left\{X: X=\mathbf{d}+Y, Y \in \mathrm{R}^{\mathrm{C}}\right\} .
$$

Thus, the expressions for the corrected likelihoods in Eqs. (4), (5) and (6) involve probabilities of the allele-count matrices $\left(\mathbf{D}\right.$ and $\left.\mathbf{D}^{(\mathrm{v})}\right)$ falling into a set. In the Eq. (4) the expression for the corrected likelihoods is the ratio between the probability of the allele count and the probability of the allele count falling into the acceptance set $\mathrm{R}^{\mathrm{C}}$. In the Eq. (5) 
the expression for the corrected likelihoods is the joint probability of the current and the previous sample divided by the probability of the allele count (in the previous sample) falling into the acceptance set $\mathrm{R}^{\mathrm{C}}$. In the Eq. (6) the expression for the corrected likelihoods is the joint probability of the current sample and the event that the previous sample falls into the acceptance set $\mathrm{R}^{\mathrm{C}}$ divided by the probability of the previous sample falling into $\mathrm{R}^{\mathrm{C}}$. An efficient way of computing the corrected likelihood is demonstrated in Subsection 2.3.

\subsection{Probability of the allele-counts falling into a set}

Claim-Consider a set

$$
S=S_{1} \times S_{2} \times \ldots \times S_{P}
$$

where $S_{i}$ is a set of allele-counts at Population $i$ at the tip of the tree; $S$ is a set of the matrix of allele-counts. Then, if we substitute $\mathrm{B}(x)_{i j}, \mathrm{~B}^{(\mathrm{L})}(x)_{i j}$ and $\mathrm{B}^{(\mathrm{R})}(x)_{i j}$ (defined in Subsection 2.1) with

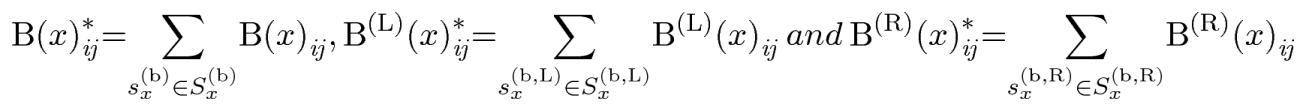

(for each node $x$ ) in the pruning algorithm, then it would yield the probability of the allelecount matrix falling into $S$. Here

$$
S_{x}^{(\mathrm{b})}=\prod_{i \in T_{x}} \mathrm{~S}_{i} ; \quad S_{x}^{(\mathrm{b}, \mathrm{L})}=\prod_{i \in T_{x}^{(\mathrm{L})}} \mathrm{S}_{i} ; \quad S_{x}^{(\mathrm{b}, \mathrm{R})}=\prod_{i \in T_{x}^{(\mathrm{R})}} \mathrm{S}_{i}
$$

$T_{x}$ is the set of all tips at or below $x ; T_{x}^{(\mathrm{L})}$ and $T_{x}^{(\mathrm{R})}$ are the sets of all tips at or below the branch coming from the left and the right sides of $x$ respectively.

We prove this in Appendix B by showing that the summations in the expression of $\mathrm{B}(x)_{i j}^{*}$, $\mathrm{B}^{(\mathrm{L})}(x)_{i j}^{*}$ and $\mathrm{B}^{(\mathrm{R})}(x)_{i j}^{*}$ can be commuted through every step of the pruning algorithm. Note that $\mathrm{B}(x)_{i j}^{*}$ is the array of probabilities (of the allele-count matrix at the tips falling into a given set) given the number of lineages and allele-counts at node $x . \mathrm{B}^{(\mathrm{L})}(x)_{i j}^{*}$ and $\mathrm{B}^{(\mathrm{R})}(x)_{i j}^{*}$ are the arrays of probabilities (of the allele-count matrix at the tips falling into a given set) given the number of lineages and allele-counts just below $x$ at the left and right branch respectively. These are same as $\mathrm{B}(x)_{i j}, \mathrm{~B}^{(\mathrm{L})}(x)_{i j}$ and $\mathrm{B}^{(\mathrm{R})}(x)_{i j}$, except that they are probabilities of a set of allele counts rather than a single value of allele count.

Note that although we have considered only the sets of the form (7) in this section, the result also holds for any other set. This is because any set can be expressed as a disjoint union of the sets of the form (7). So, the probability of the whole set can be computed as the sum over probabilities of each disjoint set. Consider a three-population tree with sample size $n$ at each population. If the rejection set 


$$
R=\left\{F_{0}^{(n)}, F_{1}^{(n)}\right\} \cup\left\{F_{n}^{(n)}, F_{n-1}^{(n)}\right\}
$$

where

$$
F_{i}^{(n)}=\left(\begin{array}{cc}
i & n-i \\
i & n-i \\
i & n-i
\end{array}\right)
$$

then

$$
\operatorname{Pr}(R)=\operatorname{Pr}\left(\left\{F_{0}^{(n)}, F_{1}^{(n)}\right\}\right)+\operatorname{Pr}\left(\left\{F_{n}^{(n)}, F_{n-1}^{(n)}\right\}\right)
$$

\section{Results}

To test the performance of our method, we performed some simulation studies. In the first study, the data consist of allele-counts in 50 haploids from each of three different populations. We have simulated data for 50 independent SNP loci. Each SNP was required to meet the criterion that its minor allele is observed to have at least two copies in the sample collected from at least one population. Thus, the rejection set

$$
R=\left\{\left\{F_{0}^{\left(n_{1}, n_{2}, n_{3}\right)}, F_{1}^{\left(n_{1}, n_{2}, n_{3}\right)}\right\}\right\} \cup\left\{\left\{F_{-0}^{\left(n_{1}, n_{2}, n_{3}\right)}, F_{-1}^{\left(n_{1}, n_{2}, n_{3}\right)}\right\}\right\}
$$

where

$$
F_{i}^{\left(n_{1}, n_{2}, n_{3}\right)}=\left(\begin{array}{cc}
i & n_{1}-i \\
i & n_{2}-i \\
i & n_{3}-i
\end{array}\right)
$$

and

$$
F_{-i}^{\left(n_{1}, n_{2}, n_{3}\right)}=\left(\begin{array}{cc}
n_{1}-i & i \\
n_{2}-i & i \\
n_{3}-i & i
\end{array}\right)
$$

The true tree is given in Figure 3(a). For the three possible tree-topologies the likelihood with no ascertainment-bias correction are given in Figure 3(b, c, \& d). The likelihoods with the ascertainment-bias correction and the corresponding MLE branch lengths of three treetopologies are given in Figure 3(e, f, \& g). As we can see from the figure, the likelihood is maximized at the correct tree-topology in all cases. However, the MLE from the corrected likelihood has branch lengths that are larger and closer to the simulation truth. This is expected because the uncorrected likelihood takes the absence of extreme alleles as evidence of short branch lengths. 
We have also tested our method for trees with four populations. We have considered a tree with four populations $\mathrm{E}, \mathrm{F}, \mathrm{G}$ and $\mathrm{H}$. The common ancestor of $\mathrm{E}$ and $\mathrm{F}$ diverge from that of $\mathrm{G}$ and $\mathrm{H}$ at the root. Then $\mathrm{E}$ and $\mathrm{F}$ diverge and also $\mathrm{G}$ and $\mathrm{H}$ diverge. Thus, there are six branches in this tree. The length of each branch is 0.02 . We have simulated 100 datasets from this tree, each with 8 haploids; for each haploid 1000 independent SNP loci are simulated. Assuming that we know the true tree-topology, we have estimated the branch lengths. The estimated biases are shown in Table 2. Using both a Wilcoxon signed-rank test and a paired t-test, we found the biases at each branch (represented as a column in Table 2) significantly different for correct vs. not corrected approach with p-values below 0.001 in all cases.

\section{Discussion}

The simulation studies indicate that as a result of not correcting for ascertainment-bias, it is possible to have significantly more bias in the branch lengths estimates for some data. Therefore, there is need for correcting for ascertainment-bias. In our examples, failure to correct for ascertainment did not affect the inference of the true tree topology.

We have introduced an efficient way of computing the probability of allele-count matrix falling in a set. We have thus improved the method of correcting for the ascertainment-bias in the pruning algorithm of RoyChoudhury et al. [9], making it faster and simpler.

Our approach is not equipped to deal with all possible ascertainment schemes. It is only designed to deal with schemes with known and deterministic ascertainment criteria. Our approach is designed for SNP data from independent loci.

Here we have used the pruning algorithm of RoyChoudhury et al. [9] to demonstrate our strategy. However, the idea of computing the probability of allele count falling into a set using pruning algorithm is more generally applicable. In particular, it is applicable to the method of Bryant et al. [1] which modifies the algorithm of RoyChoudhury et al. [9] to incorporate mutation, but keeps the structure of the algorithm and the use of the $A$ and $B$ arrays (defined in Subsection 2.1) unchanged. Our approach can also be applied to dependent loci using the composite likelihood approach of RoyChoudhury [8].

Our method of correcting for ascertainment-bias for likelihood of population trees can be applied to any data where the ascertainment strategies are well documented so that the rejection sets could be computed. In case different sets of loci in the data have different ascertainment strategies, the bias-corrected likelihood for each set may be computed separately by a separate set of runs of our method. The future applications of our method include sequence data where only some of the segregating SNPs may be selected for analysis from the whole sequence, and the ascertainment-bias correction will be needed to correct for the bias due to the selection process.

\section{Acknowledgments}

We thank Joseph Felsenstein and Robyn R. M. Gershon for their comments. This work was supported in part by National Institutes of Health program project grant GM-45344, National Institutes of Health program project grant 
GM 32544-14S1, and by National Institutes of Health Grant R01 GM071639-01A1. We also thank the anonymous reviewers for their comments.

\section{Appendix}

\section{A Steps 1 and 2}

Step 1 is derived as

$$
\begin{gathered}
\mathrm{A}^{(\mathrm{L})}(z)_{i^{\prime}}=\sum_{i=i^{\prime}}^{m_{y}^{(\mathrm{b})}} Q_{i i^{\prime}} \mathrm{A}(y)_{i}, \quad \text { (8) } \\
Q_{i i^{\prime}}=\left(\begin{array}{c}
\prod_{j=i^{\prime}+1}^{i} \lambda_{j}
\end{array}\right) \sum_{j=i^{\prime}}^{i} \frac{e^{-\lambda_{j} \tau_{y z}}}{\prod_{j^{\prime}=i^{\prime}, j^{\prime} \neq j}\left(\lambda_{j^{\prime}}-\lambda_{j}\right)}, \quad \lambda_{j}=\frac{j(j-1)}{2}, \\
\mathrm{~B}^{(\mathrm{L})}(z)_{i j}=\sum_{i^{\prime}=i j^{\prime}=0}^{m_{y}^{(\mathrm{b})}} \sum^{i^{\prime}} \mathrm{B}(y)_{i^{\prime} j^{\prime}} \frac{\beta(j, i-j)}{\beta\left(j^{\prime}, i^{\prime}-j^{\prime}\right)}\left(\begin{array}{c}
i-i^{\prime} \\
j-j^{\prime}
\end{array}\right) \frac{Q_{i i^{\prime}} \mathrm{A}(y)_{i}}{\mathrm{~A}^{(\mathrm{L})}(z)_{i^{\prime}}} .
\end{gathered}
$$

Step 2 is derived as

$$
\begin{aligned}
& \mathrm{A}(x)_{i}=\sum_{i^{\prime}=0}^{i} \mathrm{~A}^{(\mathrm{L})}(x)_{i^{\prime}} \mathrm{A}^{(\mathrm{R})}(x)_{\left(i-i^{\prime}\right)} \cdot \quad(10) \\
& \mathrm{B}(x)_{i j}=\sum_{i^{\prime}=1}^{m_{x}^{(\mathrm{b}, \mathrm{L})}} \sum_{j^{\prime}=0 j^{\prime \prime}=0}^{i^{\prime}} \sum_{i-i}^{i} \mathrm{~B}^{(\mathrm{L})}(x)_{i^{\prime} j^{\prime}} \mathrm{B}^{(\mathrm{R})}(x)_{\left(i-i^{\prime}\right) j^{\prime \prime}}\left(\begin{array}{c}
j \\
j^{\prime}
\end{array}\right)\left(\begin{array}{c}
i-j \\
i^{\prime}-j^{\prime}
\end{array}\right) /\left(\begin{array}{c}
i \\
i^{\prime}
\end{array}\right) .
\end{aligned}
$$

\section{Appendix}

\section{B Proof of the claim from Subsection 2.3}

\section{Claim}

Consider a set

$$
S=S_{1} \times S_{2} \times \ldots \times S_{P} \quad(12)
$$

where $S_{i}$ is a set of allele-counts at Population $i$ at the tip of the tree; $S$ is a set of the matrix of allele-counts. Then, if we substitute $\mathrm{B}(x)_{i j}, \mathrm{~B}^{(\mathrm{L})}(x)_{i j}$ and $\mathrm{B}^{(\mathrm{R})}(x)_{i j}$ with

$$
\mathrm{B}(x)_{i j}^{*}=\sum_{s_{x}^{(\mathrm{b})} \in S_{x}^{(\mathrm{b})}} \mathrm{B}(x)_{i j}, \mathrm{~B}^{(\mathrm{L})}(x)_{i j}^{*}=\sum_{s_{x}^{(\mathrm{b}, \mathrm{L})} \in S_{x}^{(\mathrm{b}, \mathrm{L})}} \mathrm{B}^{(\mathrm{L})}(x)_{i j} \text { and } \mathrm{B}^{(\mathrm{R})}(x)_{i j}^{*}=\sum_{s_{x}^{(\mathrm{b}, \mathrm{R})} \in S_{x}^{(\mathrm{b}, \mathrm{R})}} \mathrm{B}^{(\mathrm{R})}(x)_{i j}
$$

(for each node $x$ ) in the pruning algorithm, then it would yield the probability of the allelecount matrix falling into $S$. Here 


$$
S_{x}^{(\mathrm{b})}=\prod_{i \in T_{x}} \mathrm{~S}_{i} ; \quad S_{x}^{(\mathrm{b}, \mathrm{L})}=\prod_{i \in T_{x}^{(\mathrm{L})}} \mathrm{S}_{i} ; \quad S_{x}^{(\mathrm{b}, \mathrm{R})}=\prod_{i \in T_{x}^{(\mathrm{R})}} \mathrm{S}_{i}
$$

$T_{x}$ is the set of all tips at or below $x ; T_{x}^{(\mathrm{L})}$ and $T_{x}^{(\mathrm{R})}$ are the sets of all tips at or below the branch coming from the left and the right sides of $x$ respectively.

\section{Proof}

The array $\mathrm{B}(x)$ is an indicator function of the data for each tip $x$. Next we will prove that the $\mathrm{B}(x)^{*}$ arrays at non-tip nodes can be computed by Step 1 and Step 2 .

As before, consider a branch with nodes $z$ and $y$ at its top and bottom respectively. Without loss of generality, assume that the branch is on the left bottom side of $z$. Using Eq. (9) and noting that $S_{z}^{(\mathrm{b}, \mathrm{L})}=S_{y}^{(\mathrm{b})}$

$$
\begin{aligned}
& \mathrm{B}^{(\mathrm{L})}(z)_{i j}^{*}=\quad \sum_{s_{z}^{(\mathrm{b}, \mathrm{L})} \in S_{z}^{(\mathrm{b}, \mathrm{L})}} \operatorname{Pr}\left(s_{z}^{(\mathrm{b}, \mathrm{L})} \mid n_{x}^{(\mathrm{L})}=i, r_{x}^{(\mathrm{L})}=j\right) \\
& =\quad \sum_{s_{z}^{(\mathrm{b}, \mathrm{L})} \in S_{z}^{(\mathrm{b}, \mathrm{L})}} \sum_{i^{\prime}=i j^{\prime}=0}^{m_{y}^{(\mathrm{b})}} \sum_{j^{\prime}=0}^{i^{\prime}} \mathrm{B}(y)_{i^{\prime} j^{\prime}} \frac{\beta(j, i-j)}{\beta\left(j^{\prime}, i^{\prime}-j^{\prime}\right)}\left(\begin{array}{c}
i-i^{\prime} \\
j-j^{\prime}
\end{array}\right) \frac{Q_{i i^{\prime}} \mathrm{A}(y)_{i}}{\mathrm{~A}^{(\mathrm{L})}(z)_{i^{\prime}}}
\end{aligned}
$$

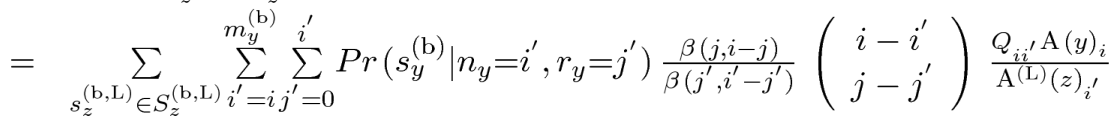

$$
\begin{aligned}
& =\sum_{i^{\prime}=i j^{\prime}=0}^{m_{y}^{(\mathrm{b})}} \sum^{i^{\prime}} \frac{\beta(j, i-j)}{\beta\left(j^{\prime}, i^{\prime}-j^{\prime}\right)}\left(\begin{array}{c}
i-i^{\prime} \\
j-j^{\prime}
\end{array}\right) \frac{Q_{i i^{\prime}} \mathrm{A}(y)_{i}}{\mathrm{~A}^{(\mathrm{L})}(z)_{i}^{\prime}} \sum_{s_{z}^{(\mathrm{b}, \mathrm{L})} \in S_{z}^{(\mathrm{b}, \mathrm{L})}} \operatorname{Pr}\left(s_{y}^{(\mathrm{b})} \mid n_{y}=i^{\prime}, r_{y}=j^{\prime}\right) \\
& =\sum_{i^{\prime}=i j^{\prime}=0}^{m_{y}^{(\mathrm{b})}} \sum_{j^{\prime}}^{i^{\prime}} \frac{\beta(j, i-j)}{\beta\left(j^{\prime}, i^{\prime}-j^{\prime}\right)}\left(\begin{array}{c}
i-i^{\prime} \\
j-j^{\prime}
\end{array}\right) \frac{Q_{i^{\prime}} \mathrm{A}(y)_{i}}{\mathrm{~A}^{(\mathrm{L})}(z)_{i^{\prime}}} \sum_{s_{y}^{(\mathrm{b})} \in S_{y}^{(\mathrm{b})}} \operatorname{Pr}\left(s_{y}^{(\mathrm{b})} \mid n_{y}=i^{\prime}, r_{y}=j^{\prime}\right) \\
& =\quad \sum_{i^{\prime}=i j^{\prime}=0}^{m_{y}^{(\mathrm{b})}} \sum^{i^{\prime}} \mathrm{B}(y)_{i^{\prime} j^{\prime}}^{*} \frac{\beta(j, i-j)}{\beta\left(j^{\prime}, i^{\prime}-j^{\prime}\right)}\left(\begin{array}{c}
i-i^{\prime} \\
j-j^{\prime}
\end{array}\right) \frac{Q_{i i^{\prime}} \mathrm{A}(y)_{i}}{\mathrm{~A}^{(\mathrm{L})}(z)_{i^{\prime}}} .
\end{aligned}
$$

Thus the same equation as in Step 1 can be used to compute $\mathrm{B}^{(\mathrm{L})}(z)_{i j}^{*}$ from $\mathrm{B}(y)_{i^{\prime} j^{\prime}}^{*}$. The array $\mathrm{B}^{(\mathrm{R})}(z)^{*}$ can be similarly computed from the bottom point of the branch coming from the right side.

Using (11) and noting the fact $S_{x}^{(\mathrm{b})}=S_{x}^{(\mathrm{b}, \mathrm{L})} \times S_{x}^{(\mathrm{b}, \mathrm{R})}$ 


$$
\begin{aligned}
& \mathrm{B}(x)_{i j}^{*}=\quad \sum_{s_{x}^{(\mathrm{b})} \in S_{x}^{(\mathrm{b})}} \operatorname{Pr}\left(s_{x}^{(\mathrm{b})} \mid n_{x}=i, r_{x}=j\right) \\
& =\quad \sum_{s_{x}^{(\mathrm{b}, \mathrm{L})} \in S_{x}^{(\mathrm{b}, \mathrm{L})} s_{x}^{(\mathrm{b}, \mathrm{R})} \in S_{x}^{(\mathrm{b}, \mathrm{R})}} \operatorname{Pr}\left(\left(S_{x}^{(\mathrm{b}, \mathrm{L})}, S_{x}^{(\mathrm{b}, \mathrm{R})}\right) \mid n_{x}=i, r_{x}=j\right)
\end{aligned}
$$

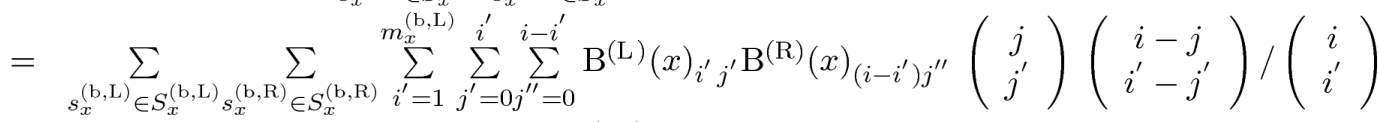

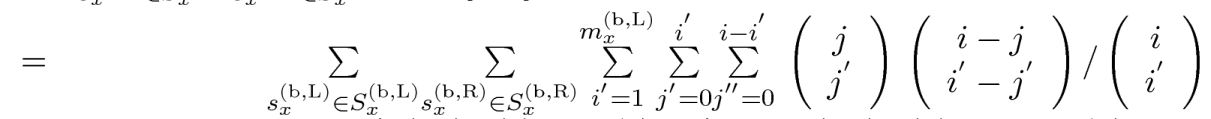

$$
\begin{aligned}
& \times \operatorname{Pr}\left(S_{x}^{(\mathrm{b}, \mathrm{L})} \mid n_{x}^{(\mathrm{L})}=i^{\prime}, r_{x}^{(\mathrm{L})}=j^{\prime}\right) \operatorname{Pr}\left(s_{x}^{(\mathrm{b}, \mathrm{R})} \mid n_{x}^{(\mathrm{R})}=i-i^{\prime}, r_{x}^{(\mathrm{R})}=j^{\prime \prime}\right) \\
& =\quad \sum_{i^{\prime}=1}^{m_{x}^{(\mathrm{b}, \mathrm{L})}} \sum_{j^{\prime}=0 j^{\prime \prime}=0}^{i^{\prime}} \sum^{i-i^{\prime}}\left(\begin{array}{c}
j \\
j^{\prime}
\end{array}\right)\left(\begin{array}{c}
i-j \\
i^{\prime}-j^{\prime}
\end{array}\right) /\left(\begin{array}{c}
i \\
i^{\prime}
\end{array}\right) \\
& \times \sum_{s_{x}^{(\mathrm{b}, \mathrm{L})} \in S_{x}^{(\mathrm{b}, \mathrm{L})}} \operatorname{Pr}\left(S_{x}^{(\mathrm{b}, \mathrm{L})} \mid n_{x}^{(\mathrm{L})}=i^{\prime}, r_{x}^{(\mathrm{L})}=j^{\prime}\right) \\
& \times \sum_{s_{x}^{(\mathrm{b}, \mathrm{R})} \in S_{x}^{(\mathrm{b}, \mathrm{R})}} \operatorname{Pr}\left(s_{x}^{(\mathrm{b}, \mathrm{R})} \mid n_{x}^{(\mathrm{R})}=i-i^{\prime}, r_{x}^{(\mathrm{R})}=j^{\prime \prime}\right) \\
& =\quad \sum_{i^{\prime}=1}^{m_{x}^{(\mathrm{b}, \mathrm{L})}} \sum_{j^{\prime}=0 j^{\prime \prime}=0}^{i^{\prime}} \sum_{i-i^{\prime}}^{i(\mathrm{~L})}(x)_{i^{\prime} j^{\prime}}^{*} \mathrm{~B}^{(\mathrm{R})}(x)_{\left(i-i^{\prime}\right) j^{\prime \prime}}^{*}\left(\begin{array}{c}
j \\
j^{\prime}
\end{array}\right)\left(\begin{array}{c}
i-j \\
i^{\prime}-j^{\prime}
\end{array}\right) /\left(\begin{array}{c}
i \\
i^{\prime}
\end{array}\right) \text {. }
\end{aligned}
$$

Thus, the same equation as in Step 2 can be used to compute $\mathrm{B}(x)^{*}$ from $\mathrm{B}^{(\mathrm{L})}(x)^{*}$ and $\mathrm{B}^{(\mathrm{R})}(x)^{*}$ as well. Repeating Steps 1 and 2 (Figure 2) we can eventually compute the arrays at the root $\left(\mathrm{A}(0)\right.$ and $\left.\mathrm{B}(0)^{*}\right)$. Then, using a equation similar to Eq. (3) we find the probability of the allele-count matrix falling into $S$ from $\mathrm{A}(0)$ and $\mathrm{B}(0)^{*}$.

\section{References}

1. Bryant, David; Remco, Bouckaert; Joseph, Felsenstein; Rosenberg, Noah A.; RoyChoudhury, Arindam. Inferring species trees directly from biallelic genetic markers: bypassing gene trees in a full coalescent analysis. 2011 arXiv.org, page arXiv:0910.4193v2.

2. Clark AG, Hubisz MJ, Bustamante CD, Williamson SH, Nielsen R. Ascertainment bias in studies of human genome-wide polymorphism. Genome Research. 2005; 15:1496-1502. [PubMed: 16251459]

3. Clayton, D. Handbook of Statistical Genetics. Vol. 2. Wiley; New York: 2003. Population Association; p. 1216-1237.

4. The International HapMap Consortium. The International HapMap Project. Nature. 2003; 426:789796. [PubMed: 14685227]

5. Elston RC. Ascertainment and age of onset in pedigree analysis. Human Heredity. 1973; 23:105112. [PubMed: 4756850]

6. Elston RC, Yelverton KC. General models for segregation analysis. Am J Hum Genet. 1975; 27:3145. [PubMed: 1171617]

7. Nielsen R, Signorovitch J. Correcting for ascertainment biases when analyzing SNP data: applications to the estimation of linkage disequilibrium. Theor Pop Biol. 2003; 63:245-255. [PubMed: 12689795]

8. RoyChoudhury A. Composite likelihood-based inferences on genetic data from dependent loci. Journal of Mathematical Biology. 201010.1007/s00285-010-0329-9

9. RoyChoudhury A, Felsenstein J, Thompson EA. A two-stage pruning algorithm for likelihood computation for a population tree. Genetics. 2008; 180:1095-1105. [PubMed: 18780754] 


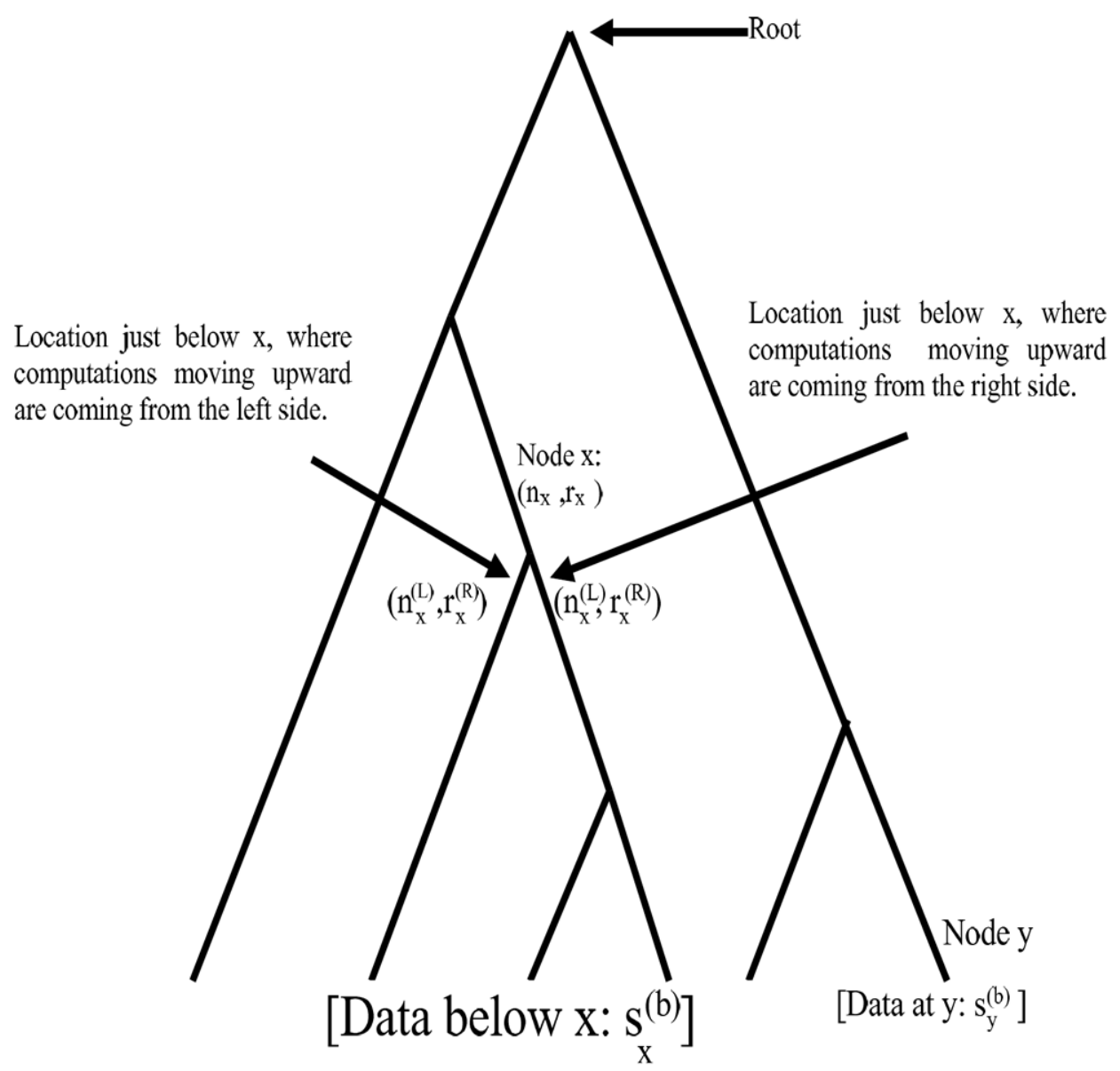

Figure 1.

Structure of an evolutionary tree 


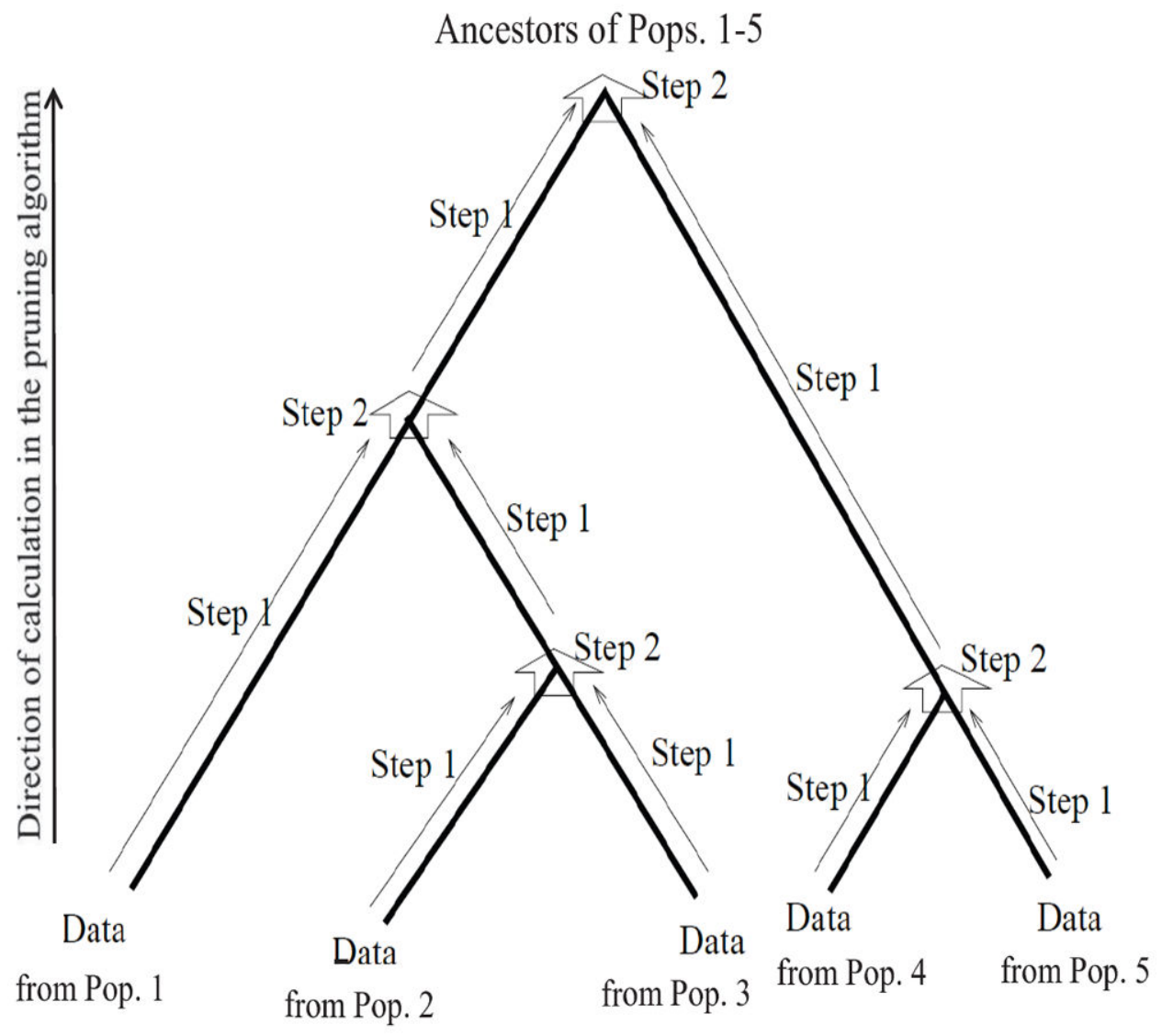

Figure 2.

The pruning algorithm works by computing two arrays of conditional probabilities at each node. The arrays at each tip are computed from the data at the tip. Then the arrays at each non-tip node are computed from the nodes directly below it. Thus, the arrays for all the nodes are computed, moving upward node by node and eventually the arrays at the root are computed. 
(a) The true tree

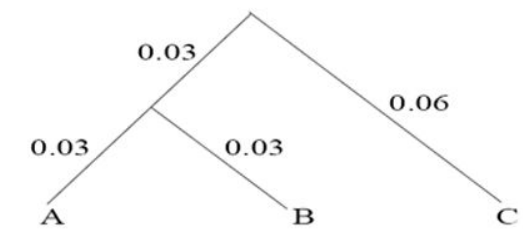

Not corrected for ascertainment

(b) Competing tree 1; no correction; MLE tree

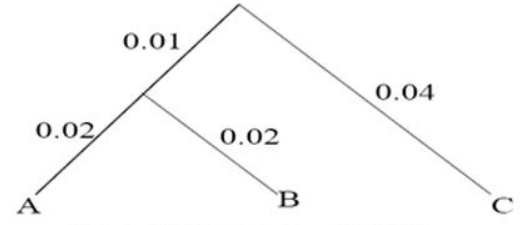

Log-likelihood: -426.50

(c) Competing tree 2; no correction

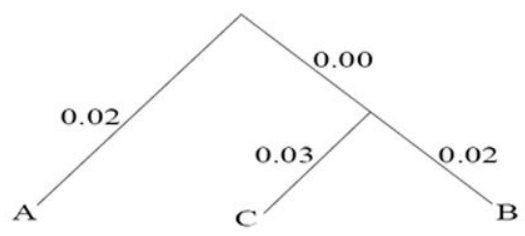

Log-likelihood: -430.06

(d) Competing tree 3 ; no correction

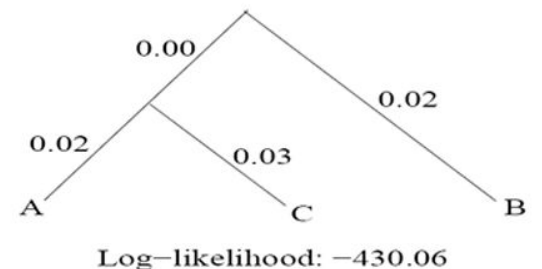

Corrected for ascertainment

(e) Competing tree 1 ; ascertainment corrected; MLE tree

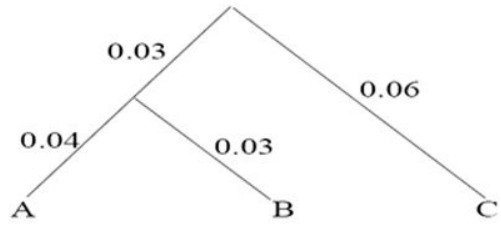

Log-likelihood: -407.95

(f) Competing tree 2; ascertainment corrected

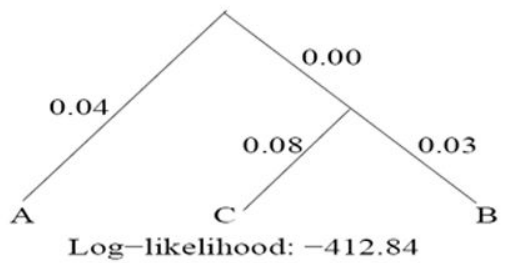

(g) Competing tree 3 ; ascertainment corrected

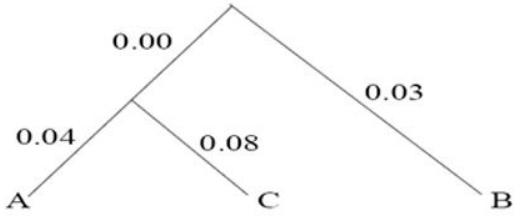

Log-likelihood: -412.84

Figure 3.

The result from the simulation study: (a) is the true tree; (b)-(d): three possible treetopologies estimated from the data not corrected for ascertainment-bias; (e)-(g): three possible tree-topologies estimated from the data corrected for ascertainment-bias. In both corrected and not-corrected cases, the likelihood is maximized at the correct tree-topology ((e) and (b)) respectively. However, the branch-length estimates have less bias when corrected for ascertainment-bias. 


\section{Table 1}

\section{Notation: see also Figure 1}

\begin{tabular}{|c|c|}
\hline$n_{x}$ & number of lineages at node $x ; n_{x} \geq 1$ \\
\hline$r_{x}$ & allele-count (the count of ' 1 ' alleles) out of $n_{x}$ lineages at node $x$; $0 \leq r_{x} \leq n_{x}$ \\
\hline$s_{x}^{(\mathrm{b})}$ & all the data at or below node $x$ \\
\hline$s_{x}^{(\mathrm{a})}$ & all the data that are not at or below node $x$ \\
\hline$m_{x}^{(\mathrm{b})}$ & total number of gene copies sampled at or below node $x ; m_{x}^{(\mathrm{b})} \geq n_{x}$ \\
\hline$m_{x}^{(\mathrm{a})}$ & total number of gene copies sampled that are not at or below node $x$ \\
\hline$n_{x}^{(\mathrm{L})}$ & number of lineages just below node $x$, in the branch coming from the left side of $x ; n_{x} \geq n_{x}^{(\mathrm{L})} \geq 1$ \\
\hline$r_{x}^{(\mathrm{L})}$ & allele-count (out of $n_{x}^{(\mathrm{L})}$ lineages) just below node $x$, in the branch coming from the left side of $x ; r_{x}^{(\mathrm{L})} \leq r_{x}$ \\
\hline$s_{x}^{(\mathrm{b}, \mathrm{L})}$ & all the data at or below the branch coming from the left side of node $x$ \\
\hline$s_{x}^{(\mathrm{a}, \mathrm{L})}$ & all the data that are not at or below the branch coming from the left side of node $x$ \\
\hline$m_{x}^{(\mathrm{b}, \mathrm{L})}$ & total number of gene copies sampled at or below the branch coming from the left side of node $x ; m_{x}^{(\mathrm{b}, \mathrm{L})} \geq n_{x}^{(\mathrm{L})}$ \\
\hline$m_{x}^{(\mathrm{a}, \mathrm{L})}$ & total number of gene copies sampled that are not at or below the branch coming from the left side of node $x$ \\
\hline$n_{x}^{(\mathrm{R})}$ & number of lineages just below node $x$, in the branch coming from the right side of $x ; n_{x} \geq n_{x}^{(\mathrm{R})} \geq 1$ \\
\hline$r_{x}^{(\mathrm{R})}$ & allele-count (out of $n_{x}^{(\mathrm{R})}$ lineages) just below node $x$, in the branch coming from the right side of $x ; r_{x}^{(\mathrm{R})} \leq r_{x}$ \\
\hline$s_{x}^{(\mathrm{b}, \mathrm{R})}$ & all the data at or below the branch coming from the right side of node $x$ \\
\hline$s_{x}^{(\mathrm{a}, \mathrm{R})}$ & all the data that are not at or below the branch coming from the right side of node $x$ \\
\hline$m_{x}^{(\mathrm{b}, \mathrm{R})}$ & total number of gene copies sampled at or below the branch coming from the right side of node $x ; m_{x}^{(\mathrm{b}, \mathrm{R})} \geq n_{x}^{(\mathrm{R})}$ \\
\hline$m_{x}^{(\mathrm{a}, \mathrm{R})}$ & total number of gene copies sampled that are not at or $x$ below the branch coming from the right side of node \\
\hline
\end{tabular}

Theor Popul Biol. Author manuscript; available in PMC 2014 October 01. 


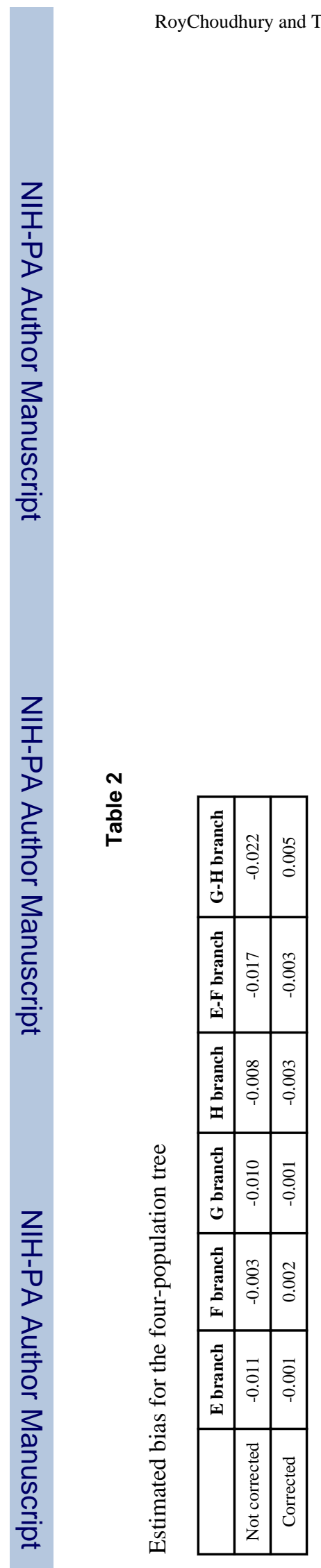

Theor Popul Biol. Author manuscript; available in PMC 2014 October 01. 